\title{
Influence of vibration amplitude on dynamic triggering of slip in sheared granular layers
}

\author{
M. Griffa, ${ }^{1, *}$ B. Ferdowsi, ${ }^{1,2}$ R. A. Guyer, ${ }^{3,4}$ E. G. Daub, ${ }^{3,5}$ P. A. Johnson, ${ }^{3}$ C. Marone,,${ }^{6,7}$ and J. Carmeliet ${ }^{1,2}$ \\ ${ }^{1}$ Swiss Federal Laboratories for Materials Science and Technology (EMPA), Überlandstrasse 129, CH-8600, Dübendorf, Switzerland \\ ${ }^{2}$ Swiss Federal Institute of Technology in Zürich (ETHZ), CH-8093, Zürich, Switzerland \\ ${ }^{3}$ Solid Earth Geophysics Group, MS D443, Los Alamos National Laboratory, Los Alamos, New Mexico 87545, USA \\ ${ }^{4}$ Department of Physics, University of Nevada, Reno, Nevada 89557, USA \\ ${ }^{5}$ Center for Nonlinear Studies, MS D443, Los Alamos National Laboratory, Los Alamos, New Mexico 87545, USA \\ ${ }^{6}$ Department of Geosciences, Pennsylvania State University, University Park, Pennsylvania 16802, USA \\ ${ }^{7}$ G3 Center and Energy Institute, Pennsylvania State University, University Park, Pennsylvania 16802, USA
}

(Received 26 September 2012; published 24 January 2013)

\begin{abstract}
We perform a systematic statistical investigation of the effect of harmonic boundary vibrations on a sheared granular layer undergoing repetitive, fully dynamic stick-slip motion. The investigation is performed using twodimensional discrete element method simulations. The main objective consists of improving the understanding of dynamic triggering of slip events in the granular layer. Here we focus on how the vibration amplitude affects the statistical properties of the triggered slip events. The results provide insight into the granular physical controls of dynamic triggering of failure in sheared granular layers.
\end{abstract}

DOI: 10.1103/PhysRevE.87.012205

PACS number(s): 45.70.Ht, 46.55.+d, 91.30.Ab

\section{INTRODUCTION}

Sheared granular layers undergoing stick-slip dynamics are broadly applied model systems for investigating earthquake physics. Sheared granular layers follow a stick-slip dynamics within certain ranges of boundary conditions and intrinsic physical features, e.g., interparticle friction coefficients. The stick-slip dynamics mimics some of the features of the earthquake dynamics. Granular layers are surrogates of the layers of rock debris located at mature fault interfaces, produced by wear and rock fragmentation and comminution due to accumulated slip on the fault. The rock debris layers are known as fault gouge. Sheared granular layers in a stickslip dynamical regime have been intensively studied in the past decade in the laboratory [1-16], by numerical simulations [17-37], and by analytical models [38-40], with the goal of improving the understanding of the granular physics role in earthquake dynamics and mechanics.

The most frequently used method for the numerical simulations of such granular systems is the discrete element method (DEM), a type of short-range molecular dynamics where the interactions among the granular layer particles are described by contact mechanics laws [41]. We report the results of 2D DEM simulations of a sheared granular layer, confined by deformable, elastic blocks, subjected to harmonic boundary vibrations. This study is an extension of our previous works that aimed at investigating the granular mechanics of slip triggering by the applied boundary ac vibration $[42,43]$. In this work we focus on an extensive statistical analysis of the stick-slip dynamics perturbation by vibration and we report the results of an investigation on the role of the vibration amplitude.

Our study is motivated by laboratory investigations performed by Savage and Marone [44] and Johnson et al. [11,16] and aims at the understanding of the physical controls of dynamic earthquake triggering. Dynamic earthquake triggering

*michele.griffa@empa.ch is induced on one fault by seismic waves radiated from an earthquake taking place on the same or another fault. The triggering may take place close to or far from the triggering earthquake. The triggered earthquake may be delayed in time (delayed triggering). Dynamic earthquake triggering is distinguished from static triggering, which consists of the redistribution of the stresses within the rupture zone following a main shock, accompanied by the triggering of many smaller earthquakes (aftershocks) [45]. The relative percentage of aftershocks that are dynamically or statically triggered is a point of considerable debate.

The discovery of dynamic earthquake triggering was made in the early 1990s due to the increase in the density of recording seismometer networks around the world, higher sensitivity instrumentation, and new data analysis methods. Its physical origins remain poorly understood and there may be a number of possible mechanisms [46].

The laboratory experiments performed by Johnson et al. with a double-direct shear apparatus and silica glass beads as model fault gouge have provided the means to investigate the role of different macroscopic scale parameters. These include the confining normal load on the sheared layer, the shear load speed, and the type of vibration (continuous or pulsed) applied to the system $[11,16]$. The experimental configuration used in those investigations does not provide access to the measurement of variables associated with the granular dynamics of the glass bead layer, while DEM simulations allow for its full characterization.

While in our previous work we focused on the mesoscopic scale granular mechanics associated with dynamic triggering of slip events, here we focus on the role of the vibration parameters, specifically on vibration amplitude, and we analyze vibration amplitude dependencies from a statistical point of view. Similar 2D and 3D studies by DEM simulations have been performed by Capozza et al. [47] and Melhus and Aranson [48]. Capozza et al. investigated the role of the boundary vibration frequency in determining an overall reduction of the macroscopic friction of the granular layer, while Melhus and Aranson studied the role of the vibration 
amplitude and frequency implementing the vibration as a fictional bulk force applied to each particle of the granular layer. Our simulation work distinguishes itself from these other works because we take into account the interplay between deformable boundary blocks and the granular layer itself, as the vibration is applied at the boundary blocks.

In Sec. II we fully describe our 2D DEM model. In Sec. III we report the procedure followed for compiling different catalogs of slip events, in the absence and in the presence of the boundary vibration. We use these catalogs for performing a statistical investigation of the dynamic triggering phenomenology in our system. The results of such statistical analysis are reported in Sec. IV. Finally, in Sec. V we analyze the obtained results and their relevance for improving the understanding of how sheared granular media respond to boundary ac perturbations and how this understanding could aid in studying dynamic earthquake triggering.

\section{DISCRETE ELEMENT METHOD MODEL}

We model a 2D sheared gouge layer with a DEM approach employing spherical particles. We present in this section each variable of the model as nondimensional, expressed in terms of respective combinations of the basic dimensional units $L_{0}=$ $150 \mu \mathrm{m}, t_{0}=1 \mathrm{~s}$, and $M_{0}=1 \mathrm{~kg}$ for length, time, and mass, respectively. Here $L_{0}$ is the largest particle radius within the overall model.

Figure 1 shows a segment of the 2D DEM model, which is composed of three groups of particles: a top driving block, a mirroring substrate block, and a granular gouge layer. The top driving block is used to confine the simulated granular gouge and to apply a shear load to it. The driving block is comprised of a set of spherical, bonded particles (top of Fig. 1) placed in two distinct layers. The top layer consists of particles with radius equal to $L_{0}$. In the simulations, these particles are driven at constant speed $V_{X, 0}=4$ in the shear direction $X$. Below this uniform layer there is a second layer, thicker than the previous one. These particles are also bonded with each other, but have distributed radii within the interval $[0.3,1.0]$. All of the particles in both layers interact with each other via radial Hookean springs that impose radial forces $F_{r}$ defined as

$$
F_{r} \equiv K_{r, b} \Delta r
$$

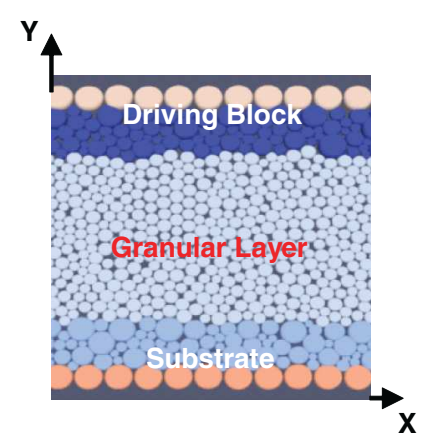

FIG. 1. (Color online) Rendering of a horizontal segment of the 2D discrete element method model. The $X$ direction corresponds to the shear load direction. A normal confining load is applied along the $Y$ direction. Periodic boundary conditions are applied along the $X$ direction. where $\Delta r$ is the difference between the interparticle distance, calculated by the centers of the two interacting particles, and the sum of the particle radii. Here $K_{r, b}=2.9775 \times 10^{7}$ is the radial compressional (tensional) spring stiffness. Its value is selected by applying a parametric study of the 2D model with the goal of obtaining a Young modulus of the particle assembly in a range typical for rocks $[9,49]$.

The substrate block (bottom of Fig. 1) is constructed similarly to the driving block and essentially mirrors it. This second set of particles is also made of a bottom layer of particles with identical radius equal to $L_{0}$. As described below, vibration is applied to the system by displacing the latter constant-radius particles along the $Y$ direction.

The driving block and the substrate are elastic assemblies representing both the laboratory and the tectonic shear blocks. Two important features distinguish them from previous DEM models of sheared granular layers.

First, the particle size distribution for the second layer of the driving block (and of the substrate) implies that the boundary between the shearing blocks and the gouge layer is characterized by a large surface roughness. This roughness is different from the one obtainable with a regular lattice of constant radius particles because it contains a variety of spatial wavelengths. Surface roughness plays a key role in determining the dynamical regime of sheared granular layers. Laboratory experiments have shown that boundary roughness plays an important role in producing stick-slip motion for granular layers composed of smooth, spherical particles. $[6,50]$. In addition, actual fault rocks are characterized by surface roughness spanning orders of magnitude in dimension [51-54].

The second important feature of the driving block and substrate as modeled here is their elastic deformability due to the system of bonds among the particles and their geometrical configuration. We implement this feature in the model to explore the dynamic interaction between the deformed granular gouge and the deformable elastic rocks. These complex interactions are a fundamental basis of earthquake physics. Only a deformable driving block provides the means for a wider spectrum of elastic energy storage and release patterns. Despite its importance, this feature has been included in only a few recently developed DEM models of sheared fault gouge $[7,9]$.

The third group of particles is the granular gouge layer, shown in the center of Fig. 1. These spherical particles are unbonded and interact with each other and with the driving block and substrate particles via repulsive-only contact forces having radial and tangential components. The radial contact force component is described by an equation similar to Eq. (1) but valid only when the interparticle distance is lower than the sum of the two interacting particles radii (overlapping particles). The radial spring stiffness is in this case $K_{r, u}=5.954 \times 10^{7}[19,55]$. The tangential component of the contact force represents the frictional force at the contact between two particles and is formulated similarly to the model proposed by Cundall and Strack [56]: At each simulation time step, the actual value of the tangential contact force is chosen as the minimum between $K_{s, u} \Delta s$ and the Coulomb threshold value $\mu F_{r}$, where $\mu$ is the friction coefficient between the two particles' surfaces. The friction 
coefficient can be either static $\mu_{s}$ or dynamic $\mu_{d}$ according to the history of the relative particle displacement. In our simulations, $\mu_{s}=\mu_{d}=0.6$. Further, $K_{s, u}=5.954 \times 10^{7}$ is the contact force tangential component's spring stiffness while $\Delta s$ is the relative displacement between the two particles along the contact direction.

The same type of packing algorithm [49] used for creating the driving block and the substrate is used for the granular layer but with radius within the interval $[0.7,1.1]$. This particle size range corresponds to the same particle size range of the glass beads used as model fault gouge in the experiments by Johnson et al. (see the section "Methods" of Ref. [11]) and leads to a quasiuniform particle size distribution [43]. Two additional damping forces, proportional to the particle translational velocity vector and to the particle angular velocity vector, respectively, are added to each particle center of mass in order to avoid the buildup of kinetic energy.

The overall model is $L_{X}=70$ long in $X$ and approximately $L_{Y}=30$ high in $Y$. Since the length-to-thickness aspect ratio of the granular layer used in the laboratory experiments by Johnson et al. [11] is too large for a reasonable simulation time, we employ periodic boundary conditions in the $X$ direction.

In the $Y$ direction, the boundary conditions are similar to the constant normal force or stress boundary condition adopted by Aharonov and Sparks [18]. A similar implementation was also adopted by Capozza et al. in their study of the effects of vibration on granular stick-slip motion [47].

Each simulation run consists of two stages. During the first stage, the consolidation stage, no shear load is imposed and the granular layer is compressed as a consequence of the vertical displacement applied to both the top of the driving block and the bottom of the substrate. The displacement continues until the normal load exerted by the granular layer is equal to $\sigma_{n}=600(\approx 4 \mathrm{MPa})$, chosen as the normal load value adopted in the laboratory experiments by Johnson et al. [11]. This consolidation stage runs for about $10^{4}$ simulation time steps, during which we observe an initial transitory, oscillatory regime for the system's thickness in $Y$, approximately exponentially decreasing towards a steady state value.

The second stage of each simulation begins after the consolidation stage and consists in keeping the normal load constant on the driving block while applying the shear load as described above. A ramp protocol is employed for gradually increasing the shear speed from 0 to $V_{X, 0}$ [42]. In the case of the perturbed runs, i.e., when boundary vibration is applied, an additional boundary condition consists in imposing a displacement in $Y$ for the substrate's bottom particles (those with equal radius $L_{0}$ ). The temporal displacement law is

$$
\begin{aligned}
u_{Y}(t)= & A \Delta t\left[\frac{\partial f}{\partial t}\left(t, t^{\prime}, T_{\nu}, \tau\right) \cos \left(\omega\left(t-t^{\prime}\right)-\frac{\pi}{2}\right)\right. \\
& \left.-\omega f\left(t, t^{\prime}, T_{\nu}, \tau\right) \sin \left(\omega\left(t-t^{\prime}\right)-\frac{\pi}{2}\right)\right]
\end{aligned}
$$

where

$$
f\left(t, t^{\prime}, T_{\nu}, \tau\right) \equiv \frac{1}{2}\left[\tanh \left(\frac{t-t^{\prime}}{\tau}\right)-\tanh \left(\frac{t-\left(t^{\prime}+T_{v}\right)}{\tau}\right)\right] .
$$

In Eqs. (2) and (3), $t=m \Delta t \forall m=0,1, \ldots$ is discretized time and $\Delta t$ is the simulation time step. Equation (2) represents a sinusoid with angular frequency $\omega=2 \pi f_{0}$, with $f_{0}=1 \mathrm{kHz}$, whose amplitude is modulated in time by a waveform with a Gaussian-like shape, given by Eq. (3). In Eq. (2), $t^{\prime}$ represents a phase shift term for centering the temporal window of ac vibration at different times during the stick-slip dynamics. In Eq. (3), $\tau=0.00125$ and $T_{v}=0.02$ are the rising and decaying time constant and width for the ac displacement waveform, respectively. In Eq. (2), $A$ is the ac vibration peak amplitude value. Equation (2) is simply derived by the first-order differentation of a temporal law for the $Y$ position of the substrate's bottom particles, equal to a cosine oscillation with amplitude modulation given by Eq. (3). We chose the Gaussian-shaped modulation in order to apply vibration smoothly, thus avoiding sharp discontinuities and associated numerical instabilities.

For the implementation of the model, we used the open source code ESyS-Particle, developed at and maintained by the Earth Systems Science Computational Centre of the University of Queensland, Brisbane, Australia. ESyS-Particle solves Newton's equations of motion for the center of mass and for the angle of rotation about the center of mass of each particle by a velocity Verlet finite difference scheme [57] and by a finite difference rotational leapfrog algorithm [58], respectively. We chose $\Delta t=25 \times 10^{-6}$ as the finite difference time step. This $\Delta t$ is small enough to guarantee numerical stability and to satisfy the sampling theorem for a vibration signal with maximum frequency $f_{\max }=2 \times 10^{5}$, which is approximately the maximum sound frequency of vibration in the laboratory experiments by Johnson et al. [11].

\section{CATALOGS OF SLIP EVENTS AND SLIP EVENT SIZE DEFINITION}

Two different types of slip event catalogs were created for this study: one with spontaneous slip events, in the absence of any boundary vibration, the others with dynamically perturbed slip events, i.e., slip events occurring in correspondence with the application of a boundary ac displacement during the stick phase preceding the slip event.

We assemble the catalog of spontaneous slip events by performing a long simulation that we call the reference run. No ac vibration is imposed during this run. The reference run consists in a long series of stick-slip events. The quantity adopted for characterizing the stick-slip dynamics is the macroscopic friction coefficient $\mu_{f}$, defined as the ratio of the macroscopic shear stress $\tau$ exerted by the granular layer on the driving block and the normal confining load $\sigma_{n}$. Figure 2(a) shows an example recorded during the reference run. Three clearly visible friction coefficient decreases, corresponding to three slip events, occur within this temporal segment, the first one about time $t=5.4$, the second one about $t=5.6$, and the third one about $t=5.8$. The reference run contains about 700 such slip events. We randomly select $N=99$ of them to create the spontaneous slip catalog. The exact onset time $t_{b, i}$ and end time $t_{e, i}$ of each $i$ th slip event, $\forall i=1, \ldots, 99$, are manually identified by selecting the local maximum and local minimum [round markers in Fig. 2(a)], respectively, within the time interval of the slip event itself. 


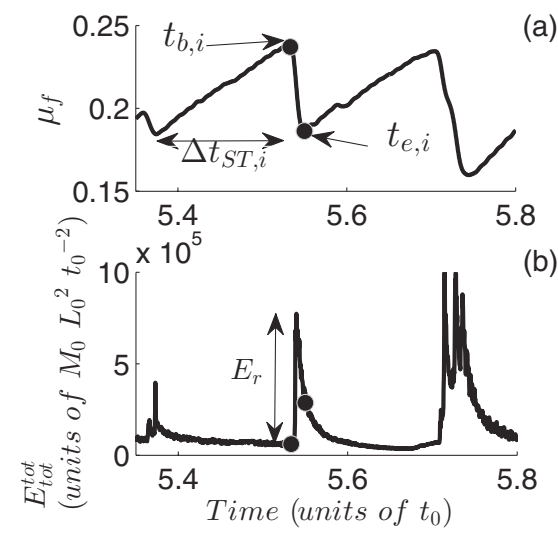

FIG. 2. Examples of stick-slip events from the spontaneous slip catalog and associated energy release. (a) Time series for the macroscopic friction coefficient $\mu_{f}$ used for identifying slip events. The round markers identify the onset $t_{b, i}$ and end $t_{e, i}$ of one slip event ( $i$ th event), defined as a decrease in the $\mu_{f}$ value. The duration of the stick period for that $i$ th event $\Delta t_{\mathrm{st}, i} \equiv t_{b, i}-t_{e, i-1}$ is highlighted by the horizontal double arrow and corresponds to one value of the time-to-failure variable. (b) Total kinetic energy of the granular layer $E_{\text {tot }}^{\text {tot }}$ for the same temporal segment in (a). The round markers identify the onset and end of the event, while the vertical double arrow indicates $E_{r}$, marking the sharp increase in $E_{\text {tot }}^{\text {tot }}$, used in this work as a proxy of the energy release during the slip event.

As a measure of the size of each slip event, we adopt a definition of energy release accompanying the event itself. First, we calculate the total kinetic energy of each $j$ th particle belonging to the granular layer $E_{j}^{\text {tot }}$. Here $E_{j}^{\text {tot }}$ is defined as

$$
E_{j}^{\mathrm{tot}} \equiv E_{j}^{\mathrm{trans}}+E_{j}^{\mathrm{rot}}
$$

where $E_{j}^{\text {trans }}$ is the $j$ th particle's translational kinetic energy and $E_{j}^{\text {rot }}$ is its rotational kinetic energy, respectively defined as

$$
E_{j}^{\text {trans }} \equiv \frac{1}{2} m_{j}\left\|\dot{\bar{u}}_{j}\right\|^{2}
$$

and

$$
E_{j}^{\mathrm{rot}} \equiv \frac{1}{2} I_{j} \omega_{j}^{2}
$$

In Eq. (5), $m_{j}$ is the $j$ th particle's mass, $\bar{u}_{j}$ is its displacement vector, an overdot indicates a temporal derivative, and $\|\cdot\|$ indicates the Euclidean norm (magnitude) of a Euclidean vector. In Eq. (6), $I_{j}$ indicates the $j$ th particle's moment of inertia and $\omega_{j}$ is its angular velocity.

We then use the total kinetic energy for the overall granular layer $E_{\text {tot }}^{\text {tot }} \equiv \sum_{j=1, \ldots, M} E_{j}^{\text {tot }}$, with $M$ the total number of granular layer's particles, as a metric for monitoring the energy release accompanying each slip event [31]. Figure 2(b) shows the $E_{\text {tot }}^{\text {tot }}$ signal for the same temporal segment of Fig. 2(a). The round markers indicate the slip event begin and end times, respectively, as in Fig. 2(a).

Figure 2(a) shows that in correspondence with the onset of the slip event (drop in the friction coefficient $\mu_{f}$ ) the total kinetic energy starts to increase sharply as a consequence of the large scale mobilization of the granular layer particles. After having achieved a local maximum value, $E_{\text {tot }}^{\text {tot }}$ starts to decrease. The slow decay continues well beyond the end of the slip event, when $\mu_{f}$ has achieved a local minimum. We observe

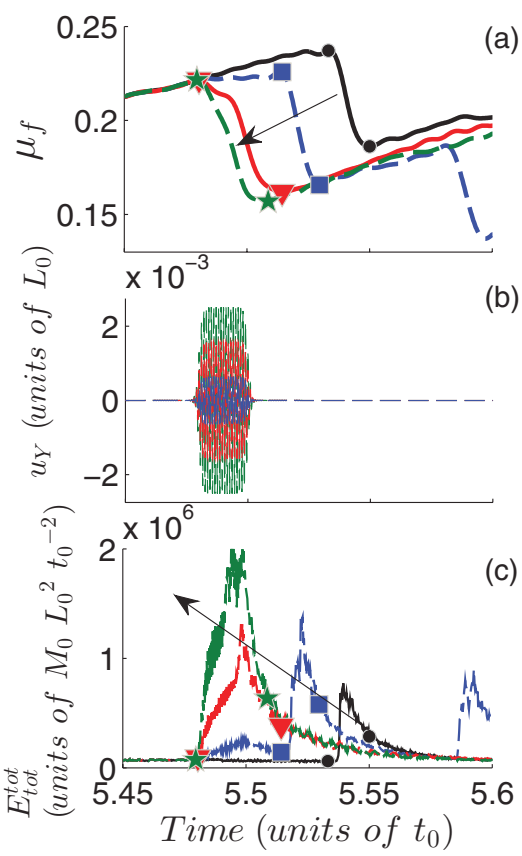

FIG. 3. (Color online) Example of dynamically triggered slip events relative to spontaneous slip events. (a) Macroscopic friction coefficient $\mu_{f}$ time series: solid black line (first from the right side) and circles, reference run, as in Fig. 2(a); dashed blue line (second from the right) and squares, corresponding perturbed run with ac vibration peak amplitude $A=0.004$; solid red line (third from right) and triangles, perturbed run with $A=0.01$; dashed green line (fourth from the right) and stars, perturbed run with $A=0.016$. The markers identify the beginning and the end of each slip event. The arrow indicates the order of curves for increasing A. (b) Boundary ac vibration displacement signal $u_{Y}$ imposed at the substrate's bottom. Curves of different colors (online) correspond to different ac vibration amplitude values as in inset (a). (c) Total kinetic energy $E_{\text {tot }}^{\text {tot }}$ signal for the granular layer. Here as well the colors (online) correspond to different ac vibration amplitude values as in (a). The markers mirror those of inset (a) for highlighting the beginning and the end of each slip event. The arrow indicates the order for increasing $A$.

this type of slow kinetic energy decrease for each slip event, both spontaneous or dynamically triggered [see Fig. 3(c)].

The variable $E_{\text {tot }}^{\text {tot }}$ is very sensitive to the granular layer's particle rearrangements since it not only captures the overall slip events captured by the $\mu_{f}$ variable but is able to depict smaller scale, clustered events that overlap and are not individually distinguishable in the $\mu_{f}$ signal, as can be seen in Figs. 2(a) and 2(b). For instance, there is one large event at about time $t=5.7 t_{0}$, visible as a large drop in $\mu_{f}$ with a small inflection point, which results in a series of three distinct, succeeding, and overlapped $E_{\text {tot }}^{\text {tot }}$ peaks.

We selected the absolute value of the difference between $E_{\text {tot }}^{\text {tot }}$ s value at the beginning of slip and its local maximum value during the slip event as a measure of the energy release $E_{r}$ associated with the event [see the vertical double arrow in Fig. 2(b)]. In this study $E_{r}$ is used as a measure of the slip event size. This measure takes into account the overall conversion of elastic potential energy into kinetic energy due to the slip event, spontaneous or dynamically triggered, and to the localized, small particle rearrangements brought by the 
boundary ac vibration and responsible for the triggering itself $[42,43]$.

In correspondence with each $i$ th slip event belonging to the spontaneous slip catalog, we conduct a simulation identical to the reference run but applying ac vibration within a time interval that starts during the stick period preceding the slip event and ends either within the stick period itself or within the slip period (perturbed run). The duration of the ac vibration is identical for each perturbed run. The center time is randomly chosen such that the maximum value of $u_{Y}$ (peak vibration displacement amplitude) is achieved approximately when $\mu_{f}$ is about $75 \%-95 \%$ of its local maximum value. For each $i$ th event of the spontaneous slip catalog, we perform ten distinct perturbed runs with different peak ac displacement amplitude $A \in$ $\{0.004,0.006,0.007,0.0085,0.009,0.01,0.012,0.014,0.016$, $0.018\}$. In this manner we obtain ten catalogs of dynamically perturbed slip events, each consisting of 99 independently perturbed events. We measure $t_{b}, t_{e}$, and $E_{r}$ for each of these 990 independent events. Figure 3(a) shows the effect of three different, increasing values of $A$ on the spontaneous slip event (black solid line, first from the right, and circles), occurring at about time $t=5.525$ [see Fig. 2(a)]. The blue dashed line, second from the right in the same plot, and the squares refer to the perturbed event for $A=0.004$. The red solid line, second from the right, and the triangles refer to the case $A=0.01$, while the green dashed line, the last from the right, and the stars refer to $A=0.016$. The markers highlight the onset and end times of the corresponding slip event for each respective vibration amplitude value. The arrow indicates how the $\mu_{f}$ signal changes with increasing $A$ value. Figure 3(b) shows the imposed ac displacement signal during the same reference stick-slip period of Fig. 3(a) and for the respective $A$ values, with corresponding colors (online), line styles, and markers. Figure 3(c) shows the total kinetic energy signal $E_{\text {tot }}^{\text {tot }}$ for the reference run and the three perturbed runs. The markers, corresponding to those of Fig. 3(a), designate the onset and end time of each event. The arrow shows how the $E_{\text {tot }}^{\text {tot }}$ signal changes with increasing $A$ values.

We remark that the increase in the peak of $E_{\text {tot }}^{\text {tot }}$ associated with the increase in the peak vibration amplitude shown in Fig. 3(c) is not incompatible with a temporal anticipation of the slip event in time [Fig. 3(a)]. A triggered event with onset before the corresponding spontaneous event takes place at a lower shear stress level. The event does not necessarily take place with a smaller conversion of elastic potential energy into kinetic energy. The available elastic potential energy, stored in a given configuration of contacts among the particles, is larger than that accumulated during a single stick period. This is supported by the fact that the shear stress steadily increases at the beginning of each run without the occurrence of stickslip dynamics and the stress drops are always smaller than that initial continuous increase. This is a typical feature of the granular stick-slip dynamics, observed in both laboratory $[2,6,12,14,59]$ and numerically simulated experiments $[27,31$, $35,60]$.

In addition, the increase in the peak value of $E_{\text {tot }}^{\text {tot }}$ with the increase in the peak vibration amplitude does not reflect the increase in the work performed by the vibration source. We performed a broader study than the one reported in this article, varying the vibration amplitude over two orders of magnitude, calculating the work done onto the system while measuring the triggered slip size using the energy release metric $E_{r}$ defined above. The energy input into the system in the form of work is always much smaller than the energy release by a factor in the interval $\left[10^{-7}, 10^{-4}\right]$.

\section{RESULTS}

\section{A. Temporal effect of vibration on slip events}

For each of the ten $A$ values applied we observe an effect on each of the 99 spontaneous slip events. The effect is a change in the stick-slip dynamics trajectory in regard to the future behavior of the friction coefficient signal $\mu_{f}$. In short, the perturbation leads to a different slip event whose onset time is in general advanced compared with the onset time of the corresponding spontaneous slip event. We call this effect dynamic triggering, a phenomenon that is documented in our previous work $[42,43]$ and in the laboratory studies by Johnson et al. [11,16]. Figure 3(a) shows that for the smallest $A$ value $[A=0.004$ (dashed blue line) $]$ the slip onset does not take place immediately with the application of the vibration displacement $u_{Y}$ [see Fig. 3(b), the blue line]: $\mu_{f}$ stops increasing and remains approximately constant for a certain time before the decrease begins. We term this type of effect on the stick-slip process a delayed dynamic triggering to distinguish from immediate triggering that takes place at larger vibration amplitudes. In the example shown in Fig. 3 the slip onset happens well after the ac vibration has achieved its maximum amplitude. However, in all cases the slip happens in advance of the spontaneous event. At larger $A$ values, the perturbed slip onset takes place immediately when vibration is applied [solid red and dashed green lines in Figs. 3(a) and 3(b)]. We term this behavior immediate dynamic triggering. Increasing $A$ above the maximum value shown, corresponding to the dashed green line in Figs. 3(a) and 3(b), does not lead to a further decrease of the onset time.

All the events comprising the perturbed slip catalogs can be included in one of these two event classes. Figure 4(b) quantifies this point by showing, for each ac vibration peak amplitude value $A$, the average (marker) and standard deviation (error bar) of the relative change in the time to failure. This variable is defined as

$$
\Gamma(A) \equiv \frac{\Delta\left(\Delta t_{\mathrm{st}}\right)}{\Delta t_{\mathrm{st}}}(A)=\frac{\Delta t_{\mathrm{st}}(A)-\Delta t_{\mathrm{st}}(0)}{\Delta t_{\mathrm{st}}(0)},
$$

where $\Delta t_{\mathrm{st}}(A)$ is the time to failure [duration of the stick period preceding an event; see Figs. 2(a) and 4(a) for examples] in a perturbed run with ac vibration peak amplitude $A$ and $\Delta t_{\mathrm{st}}(0)$ is the time to failure for the respective spontaneous slip event [Fig. 4(a)]. The average value and standard deviation of $\Gamma(A)$ were calculated over the population of 99 events, in correspondence with each $A$ value. The plot in Fig. 4(b) shows that when ac vibration is applied, the time to failure for a stickslip event on average decreases [negative value of the average of $\Gamma(A)$ ], i.e., a clock advance due to dynamic triggering. The decrease is progressively larger with larger $A$ values, i.e., with increasing $A$ values delayed triggering becomes less frequent, thus immediate triggering becomes more frequent. However, the rate of the increase in the absolute value of $\Gamma(A)$, with 

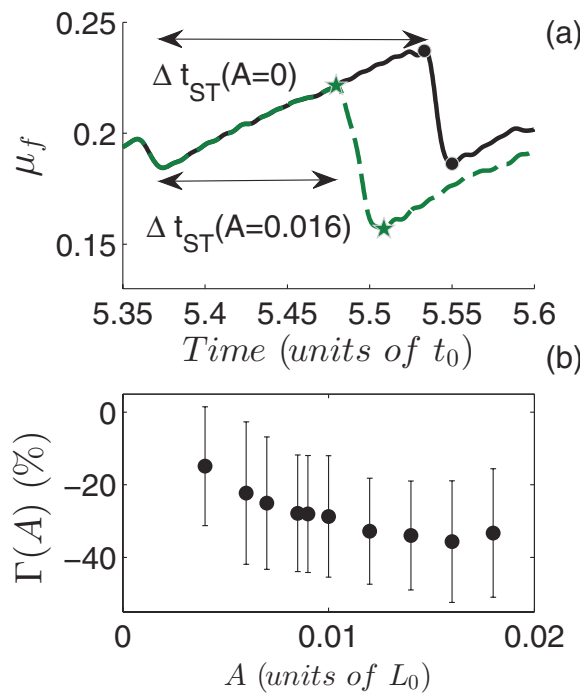

FIG. 4. (Color online) (a) Time to failure for one spontaneous slip event $\Delta t_{\mathrm{st}}(A=0)$ (solid black line and circles) [this is the same event as in Figs. 2(a) and 3(a)] and the corresponding dynamically perturbed event at the ac vibration amplitude $A=0.016, \Delta t_{\mathrm{st}}(A=$ 0.016) (dashed green line and stars). (b) Relative change $\Gamma$ in the time to failure (duration of the stick phase $\Delta t_{\mathrm{st}}$ ) with each ac vibration peak amplitude value $A$ compared with the reference run $(A=0)$. See Eq. (7) for the definition of $\Gamma(A)$. The markers indicate average values over the population of the 99 events, while the error bars indicate the standard deviation. Negative values of $\Gamma$ indicate a decrease of the time to failure in correspondence with the application of ac vibration.

increasing $A$, is larger only for the smaller $A$ values. The rate saturates for the larger values of $A$, confirming that the increase of $A$ above a certain value saturates the time advance of the slip onset. This saturation is related to the immediate triggering phenomenology.

Figure 3(c) reports the total kinetic energy $E_{\text {tot }}^{\text {tot }}$ signals corresponding to the three perturbed runs of Figs. 3(a) and 3(b). This plot helps with understanding the difference between an immediately triggered event and a delayed one. For $A=0.004$ (dashed blue line, second from right), the system undergoes a small and short-lived partial mobilization of its particles, not sufficient for immediately triggering a macroscopic event [constant $\mu_{f}$ value in Fig. 3(a)]. However, this partial mobilization nonetheless represents a significant perturbation of the stick-slip dynamics trajectory. The increase in $A$ leads to larger mobilization and anticipation of slip onset.

\section{B. Statistics of dynamically triggered slip event size at different vibration amplitude levels}

In order to investigate the effect of the boundary ac vibration on the slip size, as estimated by the energy release variable $E_{r}$ [Fig. 2(b)], we consider $E_{r}$ as a random variable, with 99 realizations corresponding to the 99 analyzed slip events, and studied its statistical features for each ac vibration peak amplitude value $A=A_{i} \forall i=1,2, \ldots, 10$ and for $A=0$ (spontaneous slip catalog).

Figure 5 shows the complementary cumulative distribution function (CCDF) of $E_{r}$ as a Weibull plot for different $A$ values. The circles refer to the spontaneous slip catalog, while the other

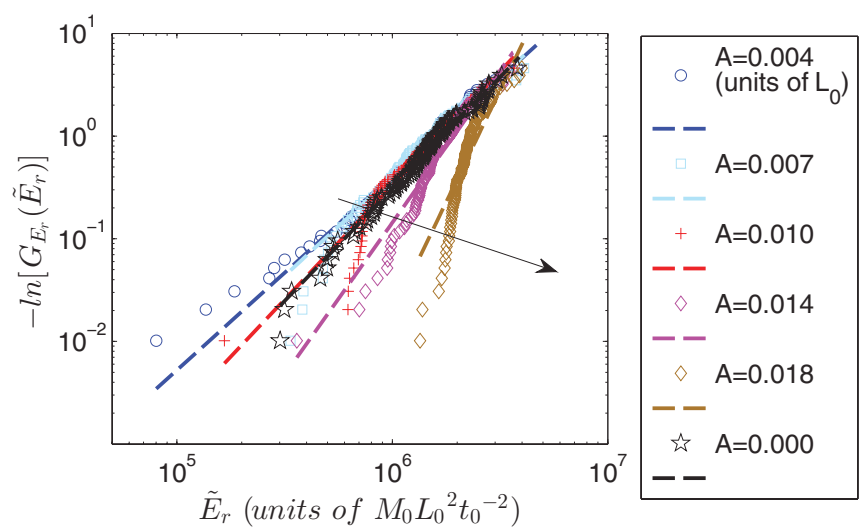

FIG. 5. (Color online) Weibull plot for the slip event energy release $E_{r}$. Different markers refer to different catalogs of slip events. The stars refer to the spontaneous slip catalog, while the other markers refer to the dynamically perturbed slip catalogs, corresponding to the values of the peak vibration amplitude value $A$. Each catalog represents the statistical ensemble for $E_{r}$ as a random variable. Here $G_{E_{r}}\left(\tilde{E}_{r}\right)$ is the complementary cumulative distribution function (CCDF) of $E_{r}$ as calculated from the corresponding catalog and it represents the probability for the event $\left\{E_{r}>\tilde{E}_{r}\right\}$ as estimated from the catalog. Each dashed line is the Weibull best fit curve of the corresponding CCDF obtained by a nonlinear least squares best fitting procedure. The arrow indicates the increasing $A$.

markers refer to the perturbed slip catalogs, different markers for different ac vibration peak amplitude values.

Here $G_{E_{r}}\left(\tilde{E}_{r}\right)$ is the probability that $E_{r}$, as a random variable, assumes a value greater than $\tilde{E}_{r}$, i.e., it is the CCDF of the random variable $E_{r}$. If a continuous random variable follows the Weibull distribution, its Weibull plot is well characterized by a line. The dashed lines in Fig. 5 are best fit curves of $G_{E_{r}}\left(\tilde{E}_{r}\right)$ according to a Weibull model function. We obtained the best fits by a nonlinear least squares procedure.

In addition to the Weibull best fit, we also performed the best fit of $G_{E_{r}}\left(\tilde{E}_{r}\right)$ with other model functions commonly used for characterizing the statistics of event size in natural systems affected by instabilities. We specifically repeated the best fit with (i) a power law model, (ii) a log-normal model, and (iii) an inverse Gaussian model [61]. In order to characterize the results of the best fits, we calculated two distinct metrics for each of them: the Kolmogorov-Smirnov distance $D_{\mathrm{KS}}$ and the root mean square error [61]. The lower the value of each statistical metric, the better the fit of the CCDF. In the case of the spontaneous slip catalog, the Weibull best fit outperforms the three other types of best fit model functions, minimizing the values of both the Kolmogorov-Smirnov distance and the root mean square error (see Table I). Daniels and Hayman reported results from laboratory experiments with $2 \mathrm{D}$ sheared granular layers, comprised of photoelastic disks, showing a strong departure from a power law CCDF for the slip event size. This was for the case of a packing fraction far away from the jamming point and in the presence of dilational boundary conditions [10]. We have similar conditions in our simulations. We did not investigate which of the two features, i.e., the possibility of dilation or compaction of the granular layer, due to the constant normal load, or the low packing fraction, plays the more significant role in determining a non-power-law 
TABLE I. Goodness of fit metrics for the best fit of the CCDF $G_{E_{r}}\left(\tilde{E}_{r}\right)$ of the slip energy release $E_{r}$ for the spontaneous slip catalog. Here $D_{\mathrm{KS}}$ denotes the Kolmogorov-Smirnov distance between the empirical CCDF and the best fit model and RMSE denotes the root mean square error of the best fit.

\begin{tabular}{lcccc}
\hline \hline Metrics & Power law & Weibull & log-normal & Inverse Gaussian \\
\hline$D_{\text {KS }}$ & 0.0884 & 0.0752 & 0.0895 & 0.1062 \\
RMSE & 0.1566 & 0.0325 & 0.0352 & 0.0437 \\
\hline \hline
\end{tabular}

CCDF for the slip event size. The slip size CCDF of our spontaneous slip catalog is thus in agreement with the results from granular stick-slip laboratory experiments performed in similar boundary or configuration conditions, but differs from the usual type of CCDF, a power law, obtained from worldwide or regional catalogs of earthquakes [62]. We observe that such catalogs include slip events occurring on different fault systems at different geographical locations or on different segments of the same fault system; thus such catalogs are not ensembles of events produced by a unique fault system, such as in the case of our modeling.

The application of ac vibration to the system during shear and the increase of its peak amplitude value $A$ lead to two main effects on the statistics of slip event size. The first effect is visible in Fig. 5: With increasing $A$ value, the CCDF of $E_{r}$, $G_{E_{r}}\left(\tilde{E}_{r}\right)$, progressively departs from a pure Weibull model.

The second effect is the most relevant one from a statistical point of view. It consists in the increase of the average value of the energy release with increasing $A$. This effect is reported in Fig. 6, where we plot the histogram of the relative difference in energy release between spontaneous slips and their respective dynamically triggered counterparts for each $A$ value. The relative change in energy release at a given vibration amplitude $A$ is calculated as

$$
\epsilon(A) \equiv \frac{\Delta E_{r}}{E_{r}}(A)=\frac{E_{r}(A)-E_{r}(A=0)}{E_{r}(A=0)},
$$

where $E_{r}(A)$ is the energy release associated with a slip event in a perturbed run at a certain vibration amplitude $A$, while $E_{r}(A=0)$ is the energy release for the corresponding spontaneous slip in the reference run.

Figure 6 shows that $\epsilon(A)$ can assume both positive and negative values, indicating an increase and decrease, respectively, of the dissipated energy during a slip event when applying ac vibration. At the lowest perturbation level $A=0.004$, the histogram is centered about 0 and is skewed towards negative values, the lowest value for $\epsilon(A)$ being approximately $-80 \%$. The skewness towards negative values indicates that several dynamically triggered slip events are smaller than their corresponding spontaneous counterparts when the vibration amplitude is low.

With increasing A, Fig. 6 shows that the center of the histogram of $\epsilon(A)$ shifts towards the right side and becomes skewed towards large positive values. At the largest $A$ values, events with up to $600 \%$ of the energy released in the reference run can occur, event sizes that seldom occur in the reference run. This means that very large vibration amplitudes can trigger rare, large slip events. The larger the $A$ value, the larger the maximum observed increase in slip event size. We remark that
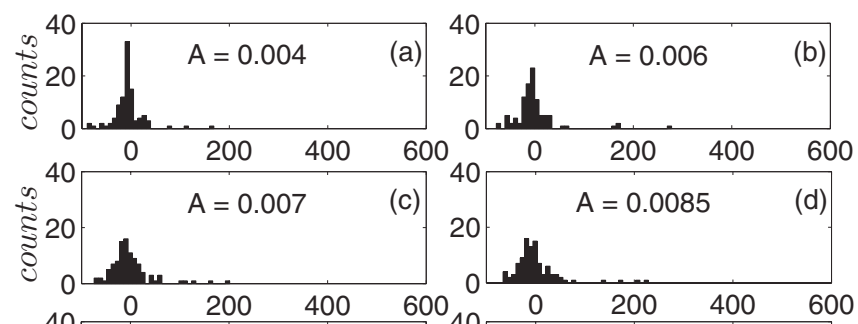

600
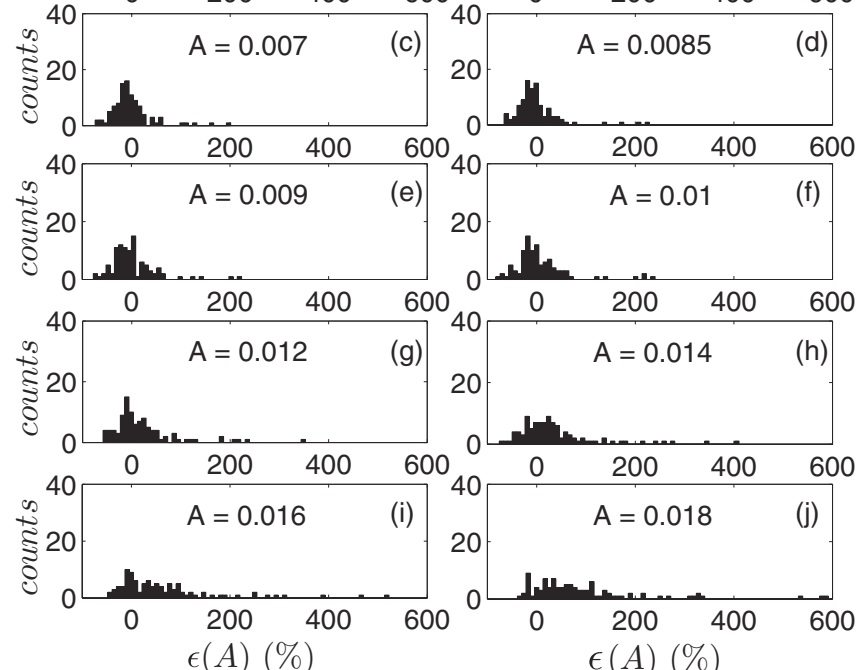

FIG. 6. Histograms for the relative change in energy release $\epsilon(A) \equiv \frac{\Delta E_{r}}{E_{r}}$ when comparing perturbed slip and spontaneous slip catalogs. Different insets refer to different ac vibration peak amplitude values: (a) $A=0.004$, (b) $A=0.006$, (c) $A=0.007$, (d) $A=$ 0.0085 , (e) $A=0.009$, (f) $A=0.01$, (g) $A=0.012$, (h) $A=0.014$, (i) $A=0.016$, and (j) $A=0.018$. With increasing $A$, on average $\epsilon$ increases and the number of dynamically triggered slip events with large energy release increases as well.

the large slip events with energy release relative change up to $600 \%$ occur in such small numbers that no statistical statement can be made about their frequency and how it changes with increase the vibration amplitude.

Figure 7 shows the average value (markers) and standard deviation (error bars) of the relative change in energy release $\epsilon(A)$ at each peak vibration amplitude value $A_{i} \forall i=1, \ldots, 10$ calculated using the population of events composing the catalogs. The average value of $\epsilon(A)$ systematically increases with $A$, especially for $A \geqslant 0.01$. The standard deviation of $\epsilon(A)$ also increases with $A$ as a consequence of the longer heavy tail towards the positive axis in correspondence with larger $A$ values.

In Fig. 8 we plot the relative difference in the energy release $\epsilon$ vs the relative difference in the time to failure $\Gamma$ for three perturbed slip catalogs at different ac vibration amplitude values. The relative difference for each variable

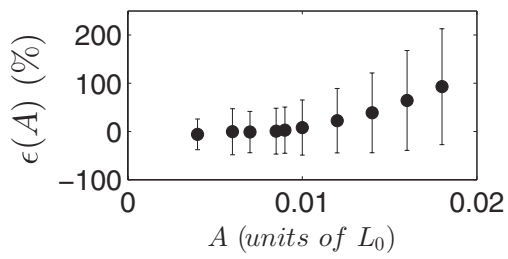

FIG. 7. Average value (marker) and standard deviation (error bar) for the relative change in slip energy release $\epsilon(A)$ for each perturbed slip catalog, i.e., in correspondence with each ac vibration peak amplitude value $A$. 


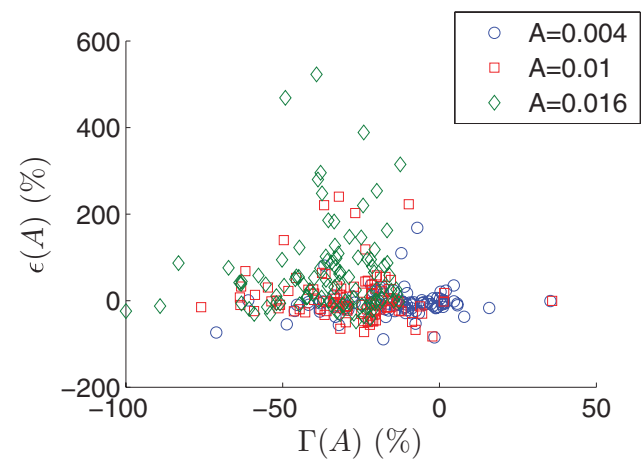

FIG. 8. (Color online) Scatter plot for the relative difference in energy release $\epsilon(A)$ vs the relative difference in time to failure $\Gamma(A)$ for each perturbed event compared with the corresponding spontaneous one. Different markers correspond to three different slip catalogs with different $A$ values.

of the plot refers to each perturbed event compared with the corresponding spontaneous one. The positive values of $\Gamma$ refer to a clock delay of the slip event, while negative values refer to a clock advance. There is not a one-to-one relation between $\Gamma$ and the amount of energy released and the plot for the three $A$ values is quite scattered.

\section{DISCUSSION AND OUTLOOK}

\section{A. Effectiveness of boundary vibration in dynamic triggering of slip events}

The investigation reported here shows that ac vibration, applied at one boundary of a sheared granular layer, systematically perturbs the spontaneous stick-slip dynamics. The perturbation is, in the majority of the cases, in the form of an anticipation of the slip event. This systematic perturbation, which we call dynamic triggering, is clearly confirmed by the results in Fig. 4(b), which shows that the time to failure $\Delta t_{\mathrm{st}}$ decreases in the presence of applied vibration. For a small number of events, in correspondence with the smallest amplitudes of vibration, the time to failure increases. These events are extreme examples of delayed dynamic triggering. Such events are observed both in the laboratory [11] and in the earth [45]).

Beyond the laboratory study performed by Johnson et al. $[11,16]$, the systematic role of applied ac vibration in anticipating slip events has been documented from a statistical point of view only by Melhus and Aranson, via 2D DEM simulations of a more simplified sheared granular layer, in the absence of deformable bounding blocks [48]. Instead of the time to failure, they monitored the shear strength of the system, i.e., the shear stress value achieved by the system at the moment of the slip onset. However, they did not report the relative change in the shear strength for the perturbed events compared with the respective spontaneous events. They provided only plots of the shear strength in correspondence with increasing values of the vibration amplitude and increasing values of the vibration frequency. They observed a decrease in the average shear strength of the system with increasing amplitude, the average being calculated from an ensemble of successive events.

A significant difference between the work of Melhus and Aranson and our work consists in the vibration implementa- tion: They applied a sinusoidal, temporally varying additional force to every particle of the granular layer, oriented in the direction orthogonal to the shear axis. In their work, they did not specify whether the prescribed harmonic force is present since the beginning of the simulation or only within a limited time interval. This approach corresponds more to introducing a bulk vibrational force due to a sort of granular temperature than to a elastic wave propagation process across the granular layer itself, as described here. Our approach is useful for investigating slip triggering by transient ac perturbations, such as seismic waves are in the case of dynamic triggering in the earth.

An additional advantage of applying a transient ac displacement to the granular layer confining boundary blocks is the possibility of selecting the time period when the perturbation begins to act. Here we limit our investigation to the case of ac vibration applied close to the onset of the spontaneous slip, when the shear stress has achieved a value larger than $75 \%$ of its maximum. This choice is guided by laboratory experiments that show triggering near the critical state is a necessity except at very low static loads [11]. A statistical investigation, similar to the one reported here, must be performed in the future by applying transient ac vibration at lower shear stress in order to achieve a broader picture of the system's response.

However, the application of a transient ac perturbation to the sheared system when it is close to its critical shear strength is relevant from a seismological point of view. It is logical that a fault is in a critical state near failure in order to be triggered. The seismic wave perturbs the failure, advancing or delaying it. The exact physical mechanism of how the dynamic shear stress increase, which is tiny compared to the potentially large stresses acting on the fault, can result in failure remains unknown. High fluid pressures, which reduce the effective stress on faults, may play a role, as dynamic triggering is commonly observed in geothermal areas where fluids are likely to be prevalent. Anisotropy in the earth's stress field may also result in low effective pressures as well.

Johnson and Jia proposed a phenomenological model to explain the onset of slip on faults close to a critical state [46]. Their model suggests that triggering may be caused by a shear modulus reduction (softening) due to the passage of seismic waves and is based on laboratory experiments [63-65]. In situ field experiments with induced ground motions have also demonstrated that seismic waves can cause material softening [66-68]. These laboratory and field experiments suggest that triggering may not be due to the very small increase in the shear stress by the propagating elastic waves, but rather may be due to the reduction in the shear modulus of the fault gouge material, leading to failure.

Having demonstrated the effectiveness of ac boundary vibration in triggering slip events, even at small vibration amplitudes, the next logical step for future work is to investigate the presence (or absence) of a decrease in our overall system's shear modulus. Our modeling approach allows such an investigation, e.g., by stopping a simulation at a time close to the onset of a dynamically triggered slip and imposing a small shear strain step $\Delta e$, recording the correspondent macroscopic shear stress $\Delta \sigma$ exerted on the boundary blocks and calculating the shear modulus $G$ as $G=\frac{\Delta \sigma}{\Delta e}[18,69]$. The same virtual test would need to be repeated several times near the slip 
onset and after it in order to track the temporal evolution of the shear modulus. In addition, the interparticle contacts and contact forces can be simultaneously tracked as well in order to obtain insight into the particle-scale mechanisms responsible for slip triggering. This type of investigation will provide more in-depth understanding of the granular physics contribution to the phenomenology of dynamic earthquake triggering at mature faults close to their critical state.

\section{B. Role of vibration amplitude in dynamic triggering of slip events}

Figure 7 summarizes one of the main results of the work presented here: An increase in the vibration amplitude systematically leads to, on average, an increase in the size of the slip events, as measured in terms of their energy release. This effect is particularly clear for peak vibration amplitude $A$ values greater than 0.01 . A comparative analysis of Fig. 8 with Fig. 4(b) also shows that for $A \geqslant 0.01$ the rate of decrease in the time to failure with increasing $A$ value decreases. Thus the increase in $A$ has its strongest effect on slip event size only for the larger values, when its effect on the triggering timing mainly consists in immediate dynamic triggering. These results are relevant for the current debate about the role of vibration amplitude in dynamic earthquake triggering, which has been the object of intense investigation in the past decade [70,71].

Our work may provide some insight into the role of large amplitude strain waves in triggering large earthquakes, the object of recent studies in relation to the observed increase of very large earthquakes in the past decade [72,73]. Several recent studies have examined whether there is temporal clustering of very large earthquakes. These studies have found mixed conclusions, with some suggesting that earthquakes cluster in time, while others find no deviation from random earthquake occurrence $[74,75]$. Dynamic triggering has been suggested as a mechanism by which large earthquakes could interact and cluster, though a recent study did not find evidence for remotely triggered large earthquakes beyond the mainshock region of the originating events [76]. These results do not exclude the possibility that large amplitude seismic waves could trigger remote, large slip events, as suggested by our results. Indeed, one important parameter influencing dynamic earthquake triggering is the relative orientation of a triggered fault system in respect to the radiation pattern of the originating earthquake: If a triggered fault is not located along the main focusing directions of the originating earthquake, the seismic waves impinging upon it will not have large amplitude.

A more in-depth understanding of the role of amplitude vibration will come from more advanced studies of both the seismological phenomenology and the associated earthquake physics. Numerical simulations such as those reported in this work may prove to be useful when considering mature faults containing fault gouge. Future investigations should focus on understanding the mechanisms of energy transfer from the boundary vibration to the granular layer itself in order to address the key question of whether the increase in slip size with vibration amplitude simply mirrors the amplitude increase itself or whether it is the effect of more complex granular rearrangements brought by vibration. This question is relevant for a better understanding of not only the physical controls of dynamic earthquake triggering on the whole earth scale but, more generally, how granular media respond to boundary dynamic perturbations, the latter being of extreme importance in local earthquake dynamic triggering, e.g., due to human activities (geotechnical drilling for the exploitation of geothermal resources or recovery of shale oil and gas by hydrofracturing, which can increase seismic activity [77]).

\section{Delayed dynamic triggering and vibration amplitude}

Delayed dynamic earthquake triggering is an even more elusive phenomenon whose physical controls remain mysterious $[78,79]$. Our results summarized in Figs. 4(b) and 8 show that low vibration amplitude may be related to delayed triggering. Laboratory experiments suggest that the rate of vibration application may also be important in regard to delayed triggering [11]. In the perturbed catalog in correspondence with the lowest vibration amplitude $(A=0.004)$ about $17 \%$ of the overall events are characterized by an increase in their time to failure if compared with the corresponding spontaneous events. The blue circles (online) in Fig. 8 show that most of these events are clustered about 0 on the time-to-failure relative change axis. However, there are a few of them characterized by a large increase in their time to failure. Figure 9 shows the friction coefficient signal for one of these delayed triggered events, for $A=0.004$ (dashed line), in comparison with the corresponding reference run signal (solid line). The vertical dashed lines show the begin and end times of the ac vibration time interval, respectively, with the peak vibration amplitude achieved about its midpoint. This perturbed event unfolds in a similar way to the one presented in Fig. 3(a), in correspondence with the same peak vibration amplitude value. In both cases (and in many others not reported here for brevity), the ac vibration induces a change in the friction coefficient signal compared with the corresponding reference run. The perturbation does not immediately trigger the slip, but nonetheless affects the system that fails later in time compared with the spontaneous event.

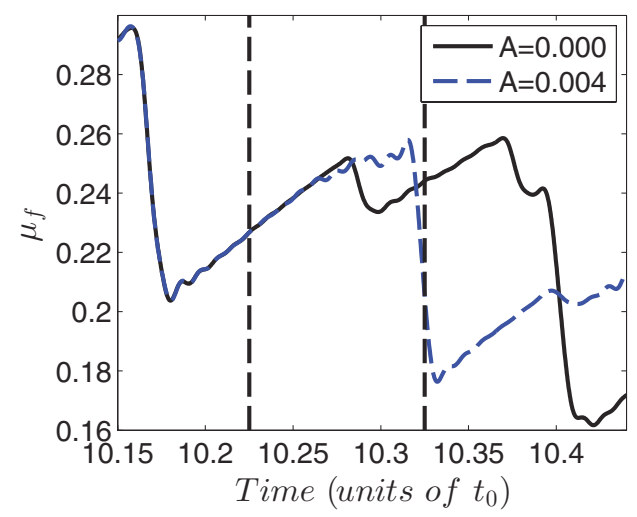

FIG. 9. (Color online) Example of delayed, dynamically triggered event, taking place later in time compared to its spontaneous counterpart: solid line, spontaneous slip event; dashed line, triggered event in correspondence with the ac vibration peak amplitude $A=$ 0.004 . The vertical dashed lines delimit the vibration time interval. The events are described by their friction coefficient signals $\mu_{f}(t)$. 
As mentioned in Sec. IV B, the delayed triggered event may be expected to happen with lower energy release compared with its spontaneous counterpart since a small amount of elastic energy associated with the deformed particles and stored at their contacts is released [see Fig. 3(c), blue line] upon applying vibration. However, as confirmed by Fig. 8, this is not the case in general: Delayed triggered events can still happen with larger energy release compared with the corresponding spontaneous events. This result confirms that even small vibration amplitude boundary perturbations can produce particle rearrangements that are small in terms of kinetic energy increase but significant from the point of view of changing the state of the system, thus the intrinsic time clock of its stick-slip dynamics.

\section{CONCLUSION}

We have performed a systematic, statistical investigation of the effects of boundary ac vibration on a confined and sheared granular layer with stick-slip dynamics. The focus of this investigation is the role of the ac vibration amplitude. The investigation was performed with a 2D discrete element method simulation approach that provides the means for modeling both the elastic blocks and the granular layer. The motivation for this study is the need for understanding the physical controls at the basis of dynamic earthquake triggering in the laboratory and at mature geologic faults, containing layers of granular materials, called fault gouge, as the result of wear and rock comminution with accumulated slip on the fault itself. Dynamic earthquake triggering is a seismological phenomenon that has been observed and characterized in the past two decades with the availability of higher density seismometer networks and more advanced instrumentation. The perturbation of the spontaneous stick-slip dynamics of a sheared granular layer by boundary vibration thus may mimic some features of the mechanics of dynamic earthquake triggering and allows for the investigation of the granular physics at its basis.

Our statistical investigation has consisted in compiling two types of slip catalogs: one catalog made of a long series of spontaneous slip events, produced by a single simulation run in the absence of any applied vibration at the boundaries of the system (spontaneous slip catalog), and a series of catalogs, each one produced by running many simulations and each simulation being identical to the reference run except for applying ac vibration during the stick period of a selected spontaneous event. The latter catalogs are called perturbed slip catalogs and differ from each other only by different values of the peak vibration amplitude, with the stick periods in each catalog corresponding with each other.

The main objectives of this work consisted in investigating what happens when vibration is applied in the region of the onset of a spontaneous slip. Having these objectives in mind, we focused on the role of the vibration amplitude as one of the key parameters of interest in dynamic triggering. Other important aspects need to be addressed in the future. Among them, we underline the role of the ac vibration in changing the temporal correlations among several events following the time of vibration application, including the question of whether or not the externally applied vibration may induce cascades of triggered events beyond perturbing a single one. In addition, similar statistical analysis should be performed also for application of vibration much earlier than the onset of a spontaneous event in order to assess the potential for dynamic triggering when the system is far away from its critical state and which parameters control and affect such potential.

We have found that boundary ac vibration systematically perturbs the spontaneous stick-slip dynamics of the system, at each vibration amplitude value, for the amplitude range investigated. The perturbation mainly consists in an anticipation of the slip onset time, compared to the onset time for the correspondent spontaneous slip event, which is analogous to dynamic triggering in the earth. The slip onset anticipation is observed to take place with the application of vibration. At lower vibration amplitude levels, the stick-slip dynamics is perturbed, but the slip onset happens a few events later than the application of vibration itself and, in a small number of events, occurring later than the spontaneous slip onset. We term this phenomenology delayed dynamic triggering, in analogy to what is observed both in the earth and in laboratory experiments with model sheared fault gouge systems [11,78-80].

We have documented in a statistically robust way the effect of increasing the vibration amplitude. Our results show that above a certain threshold, larger vibration amplitudes trigger larger size slip events, which release larger amounts of energy. The increase in vibration amplitude is also accompanied by a decrease in the time to failure of the system, although for the larger amplitude values we have observed a saturation (the time to failure cannot decrease below the initial time of vibration application). At the lower vibration amplitude values, ac vibration mostly affects the time to failure and less the size of the triggered slip events.

The slip size distribution calculated from the spontaneous slip catalog is better fit by a Weibull function than a power law, in contrast to the distributions obtained from worldwide or regional earthquake catalogs, which are usually powerlaw-like, but in agreement with catalogs of earth slip events occurring repeatedly on the same exact fault or segment of a fault system (characteristic events) [61,81]. A meaningful comparison of our results regarding the perturbation of the slip size distribution by the increasing boundary vibration amplitude should thus be performed exclusively with the results obtained from catalogs of characteristic slip events or from laboratory setups with similar boundary conditions. In both the latter cases, successive slip events happens on the same fault system, while worldwide or regional seismological catalogs include events happening on several distinct fault systems.

The results reported in this work place our previous observations on a firm statistical basis. Those observations provide an in-depth investigation of the physics of the interactions between elastic waves propagating across granular layers $[42,43]$. Future work should focus on other model and ac vibration parameters, as mentioned above, and on the role played by the dimensionality of the system (two vs three dimensions). A similar analysis in three dimensions may lead to results more easily comparable with laboratory ones about dynamically triggered slip on single fault experimental models. This work will also contribute to the broader task of understanding how sheared materials respond to perturbations and how 
instabilities and failure events can be initiated in a wide range of physical, material, engineered, and geophysical systems.

\section{ACKNOWLEDGMENTS}

We thank D. Weatherley and S. Abe for support during the implementation of our DEM model in the ESyS-Particle code and D. Passerone and C. Pignedoli for help related to the use of the high performance computing cluster Ipazia at EMPA. Our work was supported by the Swiss National Science Foundation (Projects No. 206021-128754 and No. 200021-135492) and by the LDRD Program (Institutional Support) at the Los Alamos National Laboratory, USA.
[1] C. Marone, Annu. Rev. Earth Planet. Sci. 26, 643 (1998).

[2] S. Nasuno, A. Kudrolli, A. Bak, and J. P. Gollub, Phys. Rev. E 58, 2161 (1998).

[3] K. Mair and C. Marone, J. Geophys. Res. 104, 889 (1999).

[4] F. Dalton and D. Corcoran, Phys. Rev. E 63, 061312 (2001).

[5] F. Dalton, F. Farrelly, A. Petri, L. Pietronero, L. Pitolli, and G. Pontuale, Phys. Rev. Lett. 95, 138001 (2005).

[6] J. L. Anthony and C. Marone, J. Geophys. Res. 110, 1 (2005).

[7] S. Abe and K. Mair, Geophys. Res. Lett. 32, L05305 (2005).

[8] M. Bretz, R. Zaretzki, S. B. Field, N. Mitarai, and F. Nori, Europhys. Lett. 74, 1116 (2006).

[9] K. Mair and S. Abe, Earth Planet. Sci. Lett. 274, 72 (2008).

[10] K. E. Daniels and N. W. Hayman, J. Geophys. Res. 113, B11411 (2008).

[11] P. A. Johnson, H. Savage, M. Knuth, J. Gomberg, and C. Marone, Nature (London) 451, 57 (2008).

[12] N. Higashi and I. Sumita, J. Geophys. Res. 114, B04413 (2009).

[13] N. W. Hayman, L. Ducloué, K. L. Foco, and K. E. Daniels, Pure Appl. Geophys. 168, 2239 (2011).

[14] J. Krim, P. Yu, and R. Behringer, Pure Appl. Geophys. 168, 2259 (2011).

[15] F. Leoni, A. Baldassarri, F. Dalton, A. Petri, G. Pontuale, and S. Zapperi, J. Non-Cryst. Solids 357, 749 (2011).

[16] P. A. Johnson, B. Carpenter, M. Knuth, B. Kaproth, P.-Y. Le Bas, E. Daub, and C. Marone, J. Geophys. Res. 117, B04310 (2012).

[17] D. Scott, Nature (London) 381, 592 (1996).

[18] E. Aharonov and D. Sparks, Phys. Rev. E 60, 6890 (1999).

[19] D. Place and P. Mora, J. Comput. Phys. 150, 332 (1999).

[20] J. Morgan and M. Boettcher, J. Geophys. Res. 104, 2703 (1999).

[21] J. Morgan, J. Geophys. Res. 104, 2721 (1999).

[22] J. A. Astrom, H. J. Herrmann, and J. Timonen, Phys. Rev. Lett. 84, 638 (2000).

[23] E. Aharonov and D. Sparks, Phys. Rev. E 65, 051302 (2002).

[24] J. Hazzard and K. Mair, Geophys. Res. Lett. 30, 1708 (2003).

[25] Y. Guo and J. Morgan, J. Geophys. Res. 109, B12305 (2004).

[26] Y. Guo and J. Morgan, J. Geophys. Res. 111, B12406 (2006).

[27] F. Alonso-Marroquin, I. Vardoulakis, H. J. Herrmann, D. Weatherley, and P. Mora, Phys. Rev. E 74, 031306 (2006).

[28] Y. Guo and J. Morgan, J. Geophys. Res. 112, B10403 (2007).

[29] K. Mair and J. Hazzard, Earth Planet. Sci. Lett. 259, 469 (2007).

[30] Y. Guo and J. Morgan, J. Geophys. Res. 113, B08417 (2008).

[31] A. Pena, S. McNamara, P. Lind, and H. Herrmann, Granular Matter 11, 243 (2009).

[32] T. Hatano, Geophys. Res. Lett. 36, L18304 (2009).

[33] L. Goren, E. Aharonov, D. Sparks, and R. Toussaint, J. Geophys. Res. 115, B09216 (2010).

[34] M. Pica Ciamarra, E. Lippiello, C. Godano, and L. de Arcangelis, Phys. Rev. Lett. 104, 238001 (2010).
[35] M. Pica Ciamarra, E. Lippiello, L. de Arcangelis, and C. Godano, Europhys. Lett. 95, 54002 (2011).

[36] N. Makedonska, D. Sparks, E. Aharonov, and L. Goren, J. Geophys. Res. 116, B09302 (2011).

[37] K. Mair and S. Abe, Pure Appl. Geophys. 168, 2277 (2011).

[38] K. A. Dahmen, Y. Ben-Zion, and J. T. Uhl, Phys. Rev. Lett. 102, 175501 (2009).

[39] K. Dahmen, Y. Ben-Zion, and J. Uhl, Nat. Phys. 7, 554 (2011).

[40] Y. Ben-Zion, K. Dahmen, and J. Uhl, Pure Appl. Geophys. 168, 2221 (2011).

[41] T. Poschel and T. Schwager, Computational Granular Dynamics (Springer, Berlin, 2005).

[42] M. Griffa, E. G. Daub, R. A. Guyer, P. A. Johnson, C. Marone, and J. Carmeliet, Europhys. Lett. 96, 14001 (2011).

[43] M. Griffa, B. Ferdowsi, E. Daub, R. Guyer, P. Johnson, C. Marone, and J. Carmeliet, Philos. Mag. 92, 3520 (2012).

[44] H. Savage and C. Marone, J. Geophys. Res. 113, B05302 (2008).

[45] A. Freed, Annu. Rev. Earth Planet. Sci. 33, 335 (2005).

[46] P. Johnson and X. Jia, Nature (London) 437, 871 (2005).

[47] R. Capozza, A. Vanossi, A. Vezzani, and S. Zapperi, Phys. Rev. Lett. 103, 085502 (2009).

[48] M. Melhus and I. Aranson, Granular Matter 14, 151 (2012).

[49] M. Schoepfer, S. Abe, C. Childs, and J. Walsh, Int. J. Rock Mech. Min. Sci. 46, 250 (2009).

[50] K. Mair, K. Frye, and C. Marone, J. Geophys. Res. 107, 2219 (2002).

[51] S. Brown and C. Scholz, J. Geophys. Res. 90, 12575 (1985).

[52] W. Power and T. Tullis, J. Geophys. Res. 96, 415 (1991).

[53] A. Sagy, E. Brodsky, and G. Axen, Geol. 35, 283 (2007).

[54] T. Candela, F. Renard, M. Bouchon, A. Brouste, D. Marsan, J. Schmittbuhl, and C. Voisin, Pure Appl. Geophys. 166, 1817 (2009).

[55] Y. Wang, S. Abe, S. Latham, and P. Mora, Pure Appl. Geophys. 163, 1769 (2006).

[56] P. Cundall and O. Strack, Geotechnique 29, 47 (1979).

[57] M. Allen and D. Tildesly, Computer Simulation of Liquids (Oxford Science Press, Oxford, 1987).

[58] Y. Wang, Acta Geotech. 4, 117 (2009).

[59] I. Albert, P. Tegzes, R. Albert, J. G. Sample, A.-L. Barabási, T. Vicsek, B. Kahng, and P. Schiffer, Phys. Rev. E 64, 031307 (2001)

[60] E. Aharonov and D. Sparks, J. Geophys. Res. 109, B09306 (2004).

[61] S. Abaimov, K. Tiampo, D. Turcotte, and J. Rundle, Nonlin. Processes Geophys. 16, 333 (2009).

[62] C. H. Scholz, Environmental and Engineering Geoscience (Cambridge University Press, Cambridge, 2002).

[63] R. Guyer and P. Johnson, Nonlinear Mesoscopic Elasticity (Wiley-VCH, Weinheim, 2009). 
[64] C. Inserra, V. Tournat, and V. Gusev, Appl. Phys. Lett. 92, 191916 (2008).

[65] X. Jia, T. Brunet, and J. Laurent, Phys. Rev. E 84, 020301 (2011).

[66] Z. Lawrence, P. Bodin, C. Langston, F. Pearce, J. Gomberg, P. Johnson, F.-Y. Menq, and T. Brackman, Bull. Seismol. Soc. Am. 98, 1412 (2008).

[67] Z. Lawrence, P. Bodin, and C. Langston, Bull. Seismol. Soc. Am. 99, 1650 (2009).

[68] P. Johnson, P. Bodin, J. Gomberg, F. Pearce, Z. Lawrence, and F.-Y. Menq, J. Geophys. Res. 114, B05304 (2009).

[69] D. J. Durian, Phys. Rev. Lett. 75, 4780 (1995).

[70] J. Gomberg and P. Johnson, Nature (London) 437, 830 (2005).

[71] N. van der Elst and E. Brodsky, J. Geophys. Res. 115, B07311 (2010).
[72] R. Kerr, Science 332, 411 (2011).

[73] G. Beroza, Proc. Natl. Acad. Sci. USA 109, 651 (2012).

[74] P. Shearer and P. Stark, Proc. Natl. Acad. Sci. USA 109, 717 (2012).

[75] E. Daub, E. Ben-Naim, R. Guyer, and P. Johnson, Geophys. Res. Lett. 39, L06308 (2012).

[76] T. Parsons and A. Velasco, Nat. Geosci. 4, 312 (2011).

[77] D. Giardini, Nature (London) 462, 848 (2009).

[78] D. R. Shelly, Z. Peng, D. P. Hill, and C. Aiken, Nat. Geosci. 4, 384 (2011).

[79] E. Jagla, Europhys. Lett. 93, 19001 (2011).

[80] T. Parsons, Geophys. Res. Lett. 32, L04302 (2005).

[81] S. Abaimov, D. Turcotte, and J. Rundle, Geophys. J. Int. 170, 1289 (2007). 